\section{Efficient Sampling from Truncated Bivariate Gaussians via the Box-Muller Transformation}

\section{Luca Martino, David Luengo, Joaquín Míguez}

\begin{abstract}
Many practical simulation tasks demand procedures to draw samples efficiently from multivariate truncated Gaussian distributions. In this work, we introduce a novel rejection approach, based on the Box-Muller transformation, to generate samples from a truncated bivariate Gaussian density with an arbitrary support. Furthermore, for an important class of support regions the new method allows us to achieve exact sampling, thus becoming the most efficient approach possible.
\end{abstract}

Introduction: The numerical simulation of many systems of practical interest demands the ability to produce Monte Carlo samples from truncated Gaussian distributions [5, 3, 7]. The simplest way to address this problem is to perform rejection sampling using the corresponding (non-truncated) Gaussian distribution as a proposal. This trivial method produces independent and identically distributed (i.i.d.) samples, but it is time consuming and computationally inefficient. For these two reasons, different methods have been introduced in the literature, e.g., using MCMC techniques [5, 7] or rejection sampling [1]. Unfortunately, MCMC schemes produce correlated samples, which can lead to a very slow convergence of the chain, whereas rejection methods can be computationally inefficient.

In this paper, we introduce a novel approach, based on the BoxMuller transformation (BMT) [2], to generate i.i.d. samples from truncated bivariate Gaussian distributions. The main advantages of the proposed approach are the following: (1) it allows sampling within a generic domain $\mathcal{D} \subseteq \mathbb{R}^{2}$ without any restriction and (2) the inverse transformation of the BMT maps any region $\mathcal{D} \subseteq \mathbb{R}^{2}$ (either bounded or unbounded) into a bounded region, $\mathcal{A} \subseteq \mathcal{R}=[0,1] \times[0,1]$. Hence, all the procedures developed for drawing efficiently uniform random variables within bounded regions, e.g., adaptive rejection sampling or strip methods [2, 4], can always be used. Furthermore, for an important class of support regions the BMT allows us to perform exact sampling (i.e., draw i.i.d. samples from the target distribution without any rejection), which is the most efficient situation possible.

Problem Formulation: The problem considered here is related to drawing samples from a truncated multivariate Gaussian distribution. In particular, in this letter we focus on drawing samples from a bivariate truncated standard Gaussian PDF, denoted as $\mathbf{Z} \sim T N(\mathbf{0}, \mathbf{I}, \mathcal{D})$, where the support domain $\mathcal{D} \subseteq \mathbb{R}^{2}$ is a non-null Borel set. Note that drawing samples from a non-truncated standard Gaussian distribution, $\mathbf{Z} \sim N(0, \mathbf{I})$, enables us to draw samples from an arbitrary Gaussian distribution, $\mathbf{X} \sim N(\boldsymbol{\mu}, \boldsymbol{\Sigma})$, whenever $\boldsymbol{\Sigma}$ is positive definite. More precisely, since $\boldsymbol{\Sigma}$ is positive definite, it can be expressed as $\boldsymbol{\Sigma}=\mathbf{S S}^{\top}$, using for instance the Cholesky decomposition, and the random vector $\mathbf{X}=\mathbf{S Z}+\boldsymbol{\mu}$ has the desired distribution, $\mathbf{X} \sim N(\boldsymbol{\mu}, \boldsymbol{\Sigma})$. Similarly, sampling from a truncated bivariate standard Gaussian distribution allows us to generate samples from an arbitrary truncated bivariate Gaussian. In this case, if $\mathbf{Z} \sim T N(0, \mathbf{I}, \mathcal{D})$, then we can obtain $\mathbf{X} \sim T N\left(\boldsymbol{\mu}, \boldsymbol{\Sigma}, \mathcal{D}^{*}\right)$ simply through the transformation $\mathbf{X}=\mathbf{S Z}+\boldsymbol{\mu}$, with $\boldsymbol{\Sigma}=\mathbf{S S}^{\top}$ and

$$
\mathcal{D}=\left\{\mathbf{z} \in \mathbb{R}^{2}: \mathbf{z}=\mathbf{S}^{-1}(\mathbf{x}-\mu) \quad \forall \mathbf{x} \in \mathcal{D}^{*}\right\}
$$

Box-Muller Transformation (BMT): The BMT maps a uniform random variable (RV) taking values within the rectangle $\mathcal{R}=[0,1] \times[0,1]$ into a standard Gaussian vector taking values on $\mathbb{R}^{2}$. Given two independent uniform random variables inside the interval $[0,1], U_{1}, U_{2} \sim \mathcal{U}([0,1])$, the Box-Muller transformation is given by [2]:

$$
\begin{aligned}
& Z_{1} \triangleq \mathrm{M}_{1}\left(U_{2}, U_{1}\right)=\sqrt{-2 \ln \left(U_{1}\right)} \sin \left(2 \pi U_{2}\right), \\
& Z_{2} \triangleq \mathrm{M}_{2}\left(U_{2}, U_{1}\right)=\sqrt{-2 \ln \left(U_{1}\right)} \cos \left(2 \pi U_{2}\right) .
\end{aligned}
$$

The BMT transforms the RV $\mathbf{U}=\left(U_{2}, U_{1}\right)$ into another $\mathrm{RV}, \mathbf{Z}=\left(Z_{2}, Z_{1}\right)$, following a bivariate standard Gaussian distribution, i.e., $\mathbf{Z} \sim N(\mathbf{0}, \mathbf{I})$. In the sequel, we indicate the BMT, given by (2) and (3), as $\mathrm{M}: \mathcal{R} \rightarrow \mathbb{R}^{2}$ with $\mathrm{M}\left(U_{2}, U_{1}\right)=\left(Z_{2}, Z_{1}\right)$. Hence, if we are able to draw uniformly a point $\left(u_{2}^{\prime}, u_{1}^{\prime}\right)$ in $\mathcal{R}$, we can generate a transformed point $\left(z_{2}^{\prime}, z_{1}^{\prime}\right)=\mathrm{M}\left(u_{2}^{\prime}, u_{1}^{\prime}\right)$ which is a sample from a standard bivariate Gaussian distribution.
Sampling Bivariate Truncated Gaussians via the BMT: The BMT is a continuous transformation that establishes a one-to-one correspondence between the points in $\mathcal{R}$ and the points in $\mathbb{R}^{2}$, except for the point $\left(Z_{1}=\right.$ $0, Z_{2}=0$ ). This point is obtained applying the BMT to any point with $u_{1}=1$ and $u_{2} \in[0,1]$. Thus, for any arbitrary support domain, $\mathcal{D} \subseteq \mathbb{R}^{2}$, there exists a subset, $\mathcal{A} \subseteq \mathcal{R}$, such that

$\mathcal{D}=\mathrm{M}(\mathcal{A})=\left\{\left(z_{2}, z_{1}\right) \in \mathbb{R}^{2}:\left(z_{2}, z_{1}\right)=\mathrm{M}\left(u_{2}, u_{1}\right)\right.$ with $\left.\left(u_{2}, u_{1}\right) \in \mathcal{A}\right\}$.

Consequently, for every Borel subset $\mathcal{D} \subseteq \mathbb{R}^{2}$ we can obtain $\mathcal{A} \subseteq \mathcal{R}$ as

$$
\mathcal{A} \triangleq \mathrm{M}^{-1}(\mathcal{D}) \text {. }
$$

Therefore, in order to draw a sample from a standard bivariate Gaussian restricted within a domain $\mathcal{D} \subseteq \mathbb{R}^{2}$, we can follow these two simple steps:

1) Draw a point $\mathbf{u}^{\prime}=\left(u_{2}^{\prime}, u_{1}^{\prime}\right)$ uniformly within the set $\mathcal{A}$ given by (4).

2) Transform $\mathbf{u}^{\prime}$ into a sample of the desired truncated Gaussian distribution using the BMT, i.e., $\mathbf{z}^{\prime}=\left(z_{2}^{\prime}, z_{1}^{\prime}\right)=\mathrm{M}\left(u_{2}^{\prime}, u_{1}^{\prime}\right)$.

Note that, even if $\mathcal{D}$ is unbounded, the set $\mathcal{A}$ is always bounded, since $\mathcal{A} \subseteq \mathcal{R}$, which is bounded. Therefore, an efficient procedure can always be found to perform step 1 (e.g., using rejection sampling).

As an example, consider the unbounded truncation domain $\mathcal{D}=$ $\left\{\left(z_{2}, z_{1}\right): z_{2}+z_{1}+0.9 \leq 0\right\}$, displayed in Figure 1(a). Note that the vector $\mathbf{z}=\left(z_{2}, z_{1}\right)$ can be uniquely represented by way of its polar coordinates, $r \triangleq \sqrt{z_{1}^{2}+z_{2}^{2}}$ and $\theta \triangleq \tan ^{-1}\left(z_{1} / z_{2}\right)$. These polar coordinates can be rewritten in terms of $\mathbf{u}=\left(u_{2}, u_{1}\right)$, using (2) and (3), as $r=\sqrt{-2 \ln \left(u_{1}\right)}$ and $\theta=2 \pi u_{2}$. Hence, $u_{1}=\exp \left(-\left(z_{1}^{2}+z_{2}^{2}\right) / 2\right)$ and $u_{2}=\frac{1}{2 \pi} \tan ^{-1}\left(z_{1} / z_{2}\right)$. Finally, inserting the expression for the boundary of $\mathcal{D}, z_{2}=-z_{1}-0.9$, into the equations of $u_{1}$ and $u_{2}$, we can easily obtain the contour of the bounded set $\mathcal{A}$, shown in Figure 1(b).

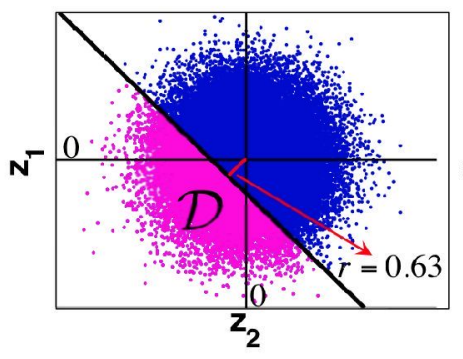

(a)

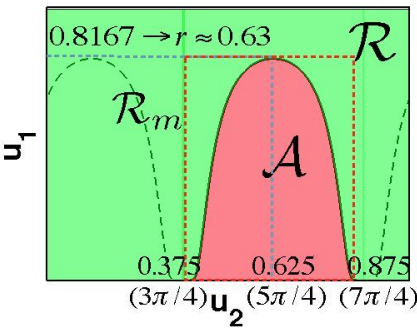

(b)
Fig. 1 (a) Example of support domain, $\mathcal{D}=\left\{\left(z_{2}, z_{1}\right): z_{2}+z_{1}+0.9 \leq 0\right\}$. We can observe that for $\left(z_{2}, z_{1}\right) \in \mathcal{D}$ we have $r \geq 0.63$ and $3 \pi / 4 \leq \theta \leq$ $7 \pi / 4$. (b) The set $\mathcal{A} \subseteq \mathcal{R}$ corresponding to $\mathcal{D}$ and the minimal rectangle $\mathcal{R}_{m}$ (dashed and dotted line) embedding $\mathcal{A} \subseteq \mathcal{R}_{m}$.

Uniform sampling inside $\mathcal{A}$ : The crucial issue for this approach is sampling uniformly inside $\mathcal{A}$. Fortunately, when the support set $\mathcal{D}$ is mapped into a simple region $\mathcal{A}$, such as a rectangle, then we can perform exact sampling (i.e., i.i.d. uniform samples can be drawn inside $\mathcal{A}$ without any rejection). This happens, for instance, for the case in which $\mathcal{D}$ is the sector of a circle delimited by the radii $r_{1}$ and $r_{2}\left(r_{1}<r_{2}\right)$ and the angles $\theta_{1}$ and $\theta_{2}\left(\theta_{1}<\theta_{2}\right)$, which includes a disk and a full circle as particular cases. The corresponding region $\mathcal{A}$ is a rectangle, i.e.,

$$
\mathcal{A}=\left\{\left(u_{2}, u_{1}\right): e^{-r_{2}^{2} / 2} \leq u_{2} \leq e^{-r_{1}^{2} / 2}, \theta_{1} /(2 \pi) \leq u_{1} \leq \theta_{2} /(2 \pi)\right\},
$$

embedded inside the unit square $\mathcal{R}$. Hence, it is straightforward to draw samples uniformly and exactly from $\mathcal{A}$ using two independent uniform random variables. Another relevant example of exact sampling occurs when the boundary of $\mathcal{D}$ is a straight line passing through the origin, i.e, $a z_{1}+b z_{2}=0$. In this case all points in $\mathcal{D}$ are completely expressed by the inequalities $r \geq 0$ and $\tan ^{-1}\{-b / a\} \leq \theta \leq \tan ^{-1}\{-b / a\}+\pi$, and $\mathcal{A}$ is again a rectangle. Additional examples of simple $\mathcal{A}$ regions where exact sampling can be performed include triangles and sectors of circles $[2,6]$.

When exact sampling cannot be performed, then we can follow the simple rejection sampling approach described in the sequel to draw uniform samples inside $\mathcal{A}$. Given a domain $\mathcal{D}$, we can find a circular sector $\mathcal{C}_{m}$ that embeds $\mathcal{D}$ (i.e., $\mathcal{D} \subseteq \mathcal{C}_{m}$ ). Now, since $\mathcal{R}_{m} \triangleq \mathrm{M}^{-1}\left(\mathcal{C}_{m}\right)$ is a rectangular region (as explained above), and since $\mathcal{R}_{m}=\mathrm{M}^{-1}\left(\mathcal{C}_{m}\right) \supseteq$ $\mathcal{A}=\mathrm{M}^{-1}(\mathcal{D})$, we can easily generate a point $\mathbf{u}^{\prime}$ uniformly inside $\mathcal{R}_{m}$, accepting it only if it belongs to $\mathcal{A}$ (or, if it is easier, we can check 
whether $\mathbf{z}^{\prime}=M\left(\mathbf{u}^{\prime}\right)$ belongs to $\mathcal{D}$ or not $)$. The acceptance probability of this approach, $p_{a}=|\mathcal{A}| /\left|\mathcal{R}_{m}\right|$ with $|\cdot|$ indicating the Lebesgue measure of a set, can be very close to one, as shown in the simulations.

Simulations: In a first simulation we use a rectangular domain with vertices $(0,0),(0, \Delta),(\Delta, 0)$ and $(\Delta, \Delta)$. In this case, we can obtain i.i.d. samples using the following rejection techniques:

- M1: Trivial rejection sampler using the non-truncated Gaussian PDF as proposal, accepting the samples that belong to $\mathcal{D}$. This is equivalent to drawing samples uniformly inside $\mathcal{R}$, accepting those that belong to $\mathcal{A}$.

- M2: Since the set $\mathcal{D}$ is bounded $(\Delta<\infty)$, we can use another trivial rejection scheme: drawing samples uniformly within $\mathcal{D}$ and accepting them with probability $\exp \left(-\left(z_{1}^{2}+z_{2}^{2}\right) / 2\right)$.

- M3: A more refined rejection procedure, introduced in [4] and discussed for truncated Gaussian PDFs in [1], consists in building the plane $y=\alpha z_{1}+\beta z_{2}+\phi$ tangent to the paraboloid $y=\left(z_{1}^{2}+z_{2}^{2}\right) / 2$ at some arbitrary point $\left(z_{1}^{*}, z_{2}^{*}\right) \in \mathcal{D}$. This induces a proposal PDF

$$
\pi\left(z_{1}, z_{2}\right) \propto \exp \left(-\alpha z_{1}-\beta z_{2}-\phi\right) \quad \forall\left(z_{1}, z_{2}\right) \in \mathcal{D},
$$

which corresponds to two truncated independent exponential PDFs. We consider two variants: the plane tangent at $\left(z_{1}^{*}, z_{2}^{*}\right)=(0,0)(\mathbf{M 3 - 1})$ and the plane tangent at $\left(z_{1}^{*}, z_{2}^{*}\right)=(\Delta / 2, \Delta / 2)(\mathbf{M} 3-2)$.

- M4: This is our rejection strategy: drawing samples from the minimal rectangle, $\mathcal{R}_{m}=\left[0, \frac{\pi}{2}\right] \times\left[1, \exp \left\{-\Delta^{2}\right\}\right]$, that covers the set $\mathcal{A}$ (i.e., $\mathcal{R}_{m} \supseteq \mathcal{A}$ ), and accepting them if they belong to $\mathcal{A}$.

We apply these four rejection techniques, computing the acceptance rate (AR), averaged over 50000 runs, as a function of the area of the domain $\mathcal{D}, \Delta^{2}$. The results are shown in Figure 2. Note that, in this case, M2 and M3-1 are equivalent. Our method, M4, provides the best performance for $\Delta^{2}>2.8$ (i.e., $\Delta>1.67$ ). Furthermore, for $\Delta \rightarrow \infty$ our technique provides virtually exact sampling (i.e., $A R \rightarrow 1$ ), whereas for M1 the AR clearly approaches $1 / 4$ and for $\mathbf{M} 2$ and $\mathbf{M} 3$ the AR $\rightarrow 0$ as $\Delta \rightarrow \infty$.

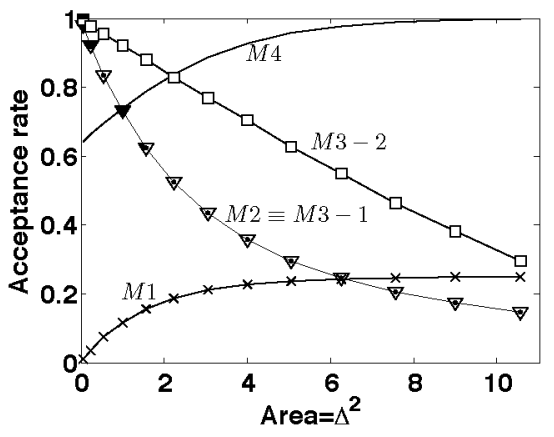

Fig. 2 Acceptance rate $(A R)$ as a function of the area of the domain $\mathcal{D}, \Delta^{2}$. M1 ( $\times$ marks), M2 $\equiv$ M3-1 (triangles), M3-2 (squares) and M4 (solid line).

As a second example, we consider $\mathcal{D}=\left\{\left(z_{2}, z_{1}\right): z_{2}+z_{1}+c \leq 0\right\}$, where $c$ is a constant parameter. In this case, we can just use the trivial strategy M1 and our method M4. M2 cannot be applied, since $\mathcal{D}$ is unbounded, and M3 is unpractical, since it is very difficult to draw samples, using a direct method, from a bivariate exponential PDF restricted to this kind of set. For $c=0$, our approach (M4) performs exact sampling (i.e., $\mathrm{AR}=1$ ), whereas $\mathrm{M} 1$ provides an $\mathrm{AR}$ of 0.5 . For $c=0.9, \mathbf{M 4}$ obtains an AR of 0.64 , whereas M1 provides an AR of 0.26 . Finally, for $c=2, \mathbf{M} 4$ obtains an AR of 0.45 , whereas M1 provides an AR of 0.12 . Hence, it can be seen that our approach M4 always outperforms M1.

Conclusions: We have proposed a new approach to draw i.i.d. samples from truncated bivariate normal distributions that allows sampling within an arbitrary domain, without any restriction. For some truncation regions, this technique provides exact sampling without any rejection. For more general cases, it allows us to develop rejection sampling approaches that can obtain better acceptance rates than state of the art methods. Furthermore, unlike most of the schemes available in the literature, our approach can always be used, regardless of whether the support is bounded or not. Future lines include extending it to arbitrary $N$-dimensional truncated Gaussian vectors.

Acknowledgment: This work has been partly financed by the Spanish government, through the DEIPRO project (TEC2009-14504-C02-01) and the CONSOLIDER-INGENIO 2010 program (CSD2008-00010).

\section{References}

[1] N. Chopin. Fast simulation of truncated Gaussian distributions. Statistics and Computing, 21(2):275-288, December 2009.

[2] L. Devroye. Non-uniform random Variate Generation. Springer, New York, 1986.

[3] A. E. Gelfand, A. F. M. Smith, and T. M. Lee. Bayesian analysis of constrained parameter and truncated data problems using Gibbs sampling. Journal of the American Statistical Association, 87:523532, March 1992.

[4] W. R. Gilks and P. Wild. Adaptive Rejection Sampling for Gibbs Sampling. Applied Statistics, 41(2):337-348, 1992.

[5] J. H. Kotecha and P. M. Djuric. Gibbs sampling approach for generation of truncated multivariate gaussian random variables. In Proc. of the 25th IEEE ICASSP, 1999.

[6] D. Luengo and L. Martino. Efficient random variable generation: ratio of uniforms and polar rejection sampling. IET Electronics Letters, 48(6):326-327, March 2012.

[7] C.P. Robert. Simulation of truncated normal variables. Statistics and Computing, 5(2):121-125, 1995. 